\title{
Lower concentration of chlorhexidine and cetyl-pyridinium chloride mouthwash demonstrates some efficacy
}

\author{
Abstracted from \\ Escribano, M. Herrera D, Morante S, Teughels W, Quirynen M, Sanz M. \\ Efficacy of a low-concentration chlorhexidine mouth rinse in non-compliant \\ periodontitis patients attending a supportive periodontal care programme: \\ a randomized clinical trial. J Clin Periodontol. 2010 Mar 37: 266-275. \\ Address for Correspondence; David Herrera, Facultad de Odontología, \\ Universidad Complutense de Madrid, Plaza Ramón y Cajal s/n, 28040 Madrid, Spain. \\ Email: davidher@odon.ucm.es
}

\section{Question: Does $0.05 \%$ chlorhexidine (CHX) and $0.05 \%$ cetyl-pyrimidine chloride(CPC) mouth rinse use in patients with moderate to severe chronic periodontitis reduce plaque and gingivitis?}

Design Randomised, parallel arm, double blind, placebo controlled trial Intervention Forty-seven non-compliant patients with inadequate oral hygiene procedures were randomly allocated into test (22 patients) and placebo (25 patients) groups. The test group received a mouthrinse containing $0.05 \% \mathrm{CHX}$ and $0.05 \% \mathrm{CPC}$ as active ingredients. The placebo rinse was identical, except that it lacked the active agents. Outcome measure Clinical, microbiological and patient centred/ adverse effect measures were taken at baseline and at 3 months. The primary outcome measures were clinical changes in plaque index (PI), measured using the modified Quigley Hein Index and changes in visual gingival inflammation (Gl). Additional clinical measures were bleeding on probing (BoP), probing pocket depth (PPD), gingival recession (GR) and clinical attachment levels (CAL). Bacterial counts for subgingival and saliva samples, frequency of detection and proportions for each bacterial species were recorded as were adverse effects and patient reported side effects. Results For the primary outcomes, there was a statistically significant $(\mathrm{P}<0.001)$ improvement in $\mathrm{Pl}$ although $\mathrm{Gl}$ showed no statistically significant difference. For the other clinical measures, there was a statically significant reduction in BoP only. For the microbiological outcomes, there were significant differences between groups for only 2 of the 9 pathogens studied. No difference in patient centred outcomes was found between the groups.

Conclusions The authors concluded that the tested mouthrinse demonstrated efficacy in reducing plaque and gingivitis, as well as decreasing the microbial load in saliva and gingival sulcus.

\section{Commentary}

This trial aimed to assess the efficacy of a low concentration chlorhexidine mouth rinse in non-compliant periodontitis patients attending a supportive periodontal programme. This mouthwash was compared with a placebo. Both the patients and clinicians were blind to treatment allocation.

In the background to the trial, the authors note that the current gold standard oral antiseptic, chlorhexidine digluconate (CHX), has numerous drawbacks for patients - namely 'tooth staining, burning feeling and soft tissue irritation'. These symptoms have been found to be dosage dependent, progressing upon concentrations above $0.1 \% .{ }^{1}$ Certainly a more efficacious and effective mouth rinse with fewer side effects would be welcomed by both patients and the profession alike. With this in mind, a lower concentration of chlorhexidine mouth rinse, $0.05 \% \mathrm{CHX}$ with cetyl-pyridinium chloride, another antimicrobial agent, was tested.

The trial's primary clinical outcomes included plaque and gingival indices. Escribano et al report a statistically significant inter group difference $(\mathrm{p}<0.001)$ in the reduction of plaque levels produced by the new mouthwash when compared to a placebo.

As ever, whilst considering the interpretation of this statistically significant difference, clinicians should consider whether this result would be clinically significant for their own patients. The trial was conducted between two secondary care centres, and a number of methodological and statistical issues should be considered whilst contemplating the generalisability of the results presented.

The patient centred and microbiological outcomes were only reported for one of the centres (Madrid), but the authors did not explain why the other centre's results were not included/provided. More patients using the new mouth rinse reported symptoms of 'tooth staining' and 'burning of the mouth'; the differences between the mouth rinses tended towards statistical significance $(\mathrm{p}=0.07$ and 0.08 respectively). Unfortunately the authors did not adequately address this issue, (which seemed to be what created their clinical question in the first place), in the discussion.

Numerous other primary and secondary outcomes were reported, with several statistical tests. It is worth considering that where sufficient analyses are carried out, the difference found could simply be the result of the play of chance rather than a true statistical difference between the interventions. For example, in a study where the statistically significant level is taken to be 0.05 i.e. 1 in 20 , then 1 out of 20 tests carried out are likely to show significance just through chance. Over 40 statistical tests were carried out for 'microbiological outcome variables - subgingival samples' alone, and from this only 
Fusobacterium nucleatum and Prevotella intermedia showed statistically significant reduction in subgingival counts. With the number of tests carried out, one would consider whether these statistical significance differences are merely the result of chance.

It is important that clinical researchers consider the likely end users of their research. It is therefore commendable that Escribano et al included smokers in this trial as a proportion of patients who suffer from moderate to severe periodontitis would also be likely to smoke. Unfortunately any interpretation of the data for these included patients is complicated by the fact that those who smoke less than 10 cigarettes daily were still included in the non-smoker group.

This trial aimed to assess the efficacy of this new mouth rinse, and the authors report positive findings for reduction in plaque and gingivitis as well as a decline in salivary and gingival sulcus microbial load. In the background to the trial, the authors stated a desire to test the new mouthwash due to the gold standard CHX shortcom- ings in patient centred outcomes eg. tooth staining etc. This study demonstrated that patients receiving this new mouth wash continued to suffer from these symptoms and did not adequately address this issue in their discussion. Regarding translation of the findings to patient care and use of a reduced concentration CHX, cautious interpretation is advised. Follow on research looking at the actual effectiveness of this new mouth wash compared with the old CHX one would be welcomed.

Thomas Lamont

Dental Health Services Research Unit, Dundee Dental School, Dundee, Scotland

1. Smith RG, Moran J, Addy M, Doherty F, Newcombe RG. Comparative staining in vitro and plaque inhibitory properties in vivo of $0.12 \%$ and $0.2 \%$ chlorhexidine mouthrinses. J Clin Periodontol. 1995; 22: 613-617.

Evidence-Based Dentistry (2012) 13, 52-53. doi:10.1038/sj.ebd.6400862 